\title{
Nefrotossicità
}

\section{dei mezzi di contrasto iodati}

\author{
S. Tazzari ${ }^{2}$, M. Meroni ${ }^{1}$, L. Torri Tarelli ${ }^{2}$, A. Sessa ${ }^{1}$ \\ ${ }^{I}$ Servizio di Nefrologia e Dialisi, Ospedale di Vimercate \\ ${ }^{2}$ Anatomia Umana Normale, Università di Milano
}

mezzi di contrasto usati nelle metodiche diagnostiche di radiologia, a causa delle dosi impiegate, talvolta molto elevate, debbono soddisfare importanti esigenze che, oltre la opacizzazione ottimale degli organi sottoposti a studio contrastografico, sono la massima tollerabilità unita alla eliminazione più rapida possibile dall'organismo.

Le sostanze contrastografiche vengono distinte in due gruppi, mezzi negativi e positivi, relativamente alle loro proprietà fisiche.

I mezzi di contrasto negativi, o trasparenti, fondano le loro caratteristiche fisiche sul minore assorbimento di raggi $\mathrm{X}$ rispetto all'acqua (maggiore costituente del corpo umano) ed alla più parte dei sali, per cui l'organo da essi circondato o riempito, risalta rispetto alle strutture circonvicine. È il caso, per esempio, di formazioni che già possiedono un contrasto trasparente naturale, come la bolla gastrica, i seni nasali e paranasali, la laringe, la trachea e i polmoni.

I mezzi di contrasto positivi, o radioopachi, a differenza dei precedenti, presentano la caratteristica di essere meno permeabili ai raggi $X$ rispetto alle strutture anatomiche e possiedono proprietà fondamentali quali: un elevato numero atomico per poter essere sufficientemente radioopachi, una scarsa o nulla tossicità per ingestione o iniezione ed, infine, un'attività il più possibile fisiologica, per non alterare la normale funzione dell'organo o dell'apparato esplorato. Sono distinti in:

A - Solfato di Bario. Da almeno 50 anni rappresenta il classico opacizzante dell'apparato digerente per relativa inerzia, buona radioopacità, non assorbibilità ed idroinsolubilità.

B - Composti iodati in sospensioni oleose. Vengono usati per prolungare la concentrazione locale di mezzo di contrasto e trovano impiego nella broncografia e nella linfografia. Presentano un'elevata concentrazione di Iodio (più di $500 \mathrm{mg} / \mathrm{ml}$ ), una bassa viscosità, un elevato peso specifico ed una elevata tensione superficiale. Non vengono eliminati dall'organismo, ma vi permangono a lungo.

C - Composti iodati idrosolubili. Rappresentano la gran parte di tutte le preparazioni contrastografiche a base di Iodio impiegate per lo studio morfologico e funzionale di vie renali e biliari, vasi arteriosi e venosi, cavità cardiache, per rinforzo nella tomografia assiale computerizzata, per artografia, colangiografia percutanea transepatica, colangiografia pre e post operatoria, controllo della posizione di cateteri vascolari, isterosalpingografia, ed ancora molte altre tecniche.

I moderni mezzi di contrasto organoio- dati hanno come struttura di base l'anello benzenico che funge da trasportatore stabile di tre atomi di Iodio con una funzione acida che consente la preparazione di sali solubili in acqua; l'inserimento nella molecola di gruppi chimici ne migliora la solubilità e le conferisce un alto potere contrastografico, un'elevata solubilità e, non ultima caratteristica, una scarsa tossicità. Il problema relativo all'iperosmolarità, caratteristica negativa dei mezzi di contrasto, è stato risolto attraverso la sintesi di sali di dimeri monoacidi (Ioxaglati di sodio e meglumina) e la trasformazione del gruppo carbossilico salificato del mezzo di contrasto convenzionale (che in soluzione ionizza) in un gruppo che non si dissocia, cioè un amide (-CONH-R).

Da queste manipolazioni strutturali sono derivati i mezzi di contrasto non ionici monomeri a bassa osmolarità quali Metrazimide, Iopamidolo, Iohexol, Iosimide, Ioprimide e dimeri come lo Iotriolo.

I parametri organici che interessano per stabilire i livelli di interferenza di queste sostanze con l'organismo sono sostanzialmente due: la viscosità e l'osmolarità.

1) La viscosità delle soluzioni dei mezzi di contrasto, correlata alla concentrazione ed alle dimensioni delle particelle, dipende da numerosi fattori quali: la TEMPERATURA (la viscosità diminuisce 
con l'aumentare della temperatura), la CONCENTRAZIONE del soluto (la viscosità aumenta con l'aumentare del contenuto di Iodio o con l'aumentare della concentrazione di Iodio), la NATURA DELLE PARTICELLE presenti nella soluzione (poiché la viscosità è un indice della frizione interna di un liquido, ne deriva che la viscosità aumenta con l'aumentare delle đimensioni delle particelle). Se si confronta la viscosità delle soluzioni saline pure, si osserva che essa aumenta dalle soluzioni di sale di Sodio a quelle di sale di Meglumina a causá delle maggiori dimensioni del catione megluminico, ammettendo che 1'anione acido radioopaco sia il medesimo per entrambe le soluzioni.

2) Analoga importanza riveste l'osmolarità del mezzo di contrasto. Le particelle (molecole, ioni) in soluzione si trovano in condizione analoga alle molecole di un gas. Queste ultime, com'è noto, tendono ad occupare tutto lo spazio a loro disposizione e, analogamente, una soluzione concentrata posta al fondo di un recipiente sotto uno strato di soluzione meno concentrata, con lo stesso solvente, tende per diffusione a dare una soluzione omogenea. Per quanto riguarda l'osmolarità dei mezzi di contrasto, i liquidi corporei possono essere divisi essenzialmente in due compartimenti: extracellulare ed intracellulare.

Nell'ambito del liquido extracellulare vengono compresi il plasma sanguigno ed il liquido interstiziale, cioè quel liquido che si trova fuori dal sistema vascolare e bagna le cellule. I vari compartimenti liquidi sono diversi per composizione e distribuzione degli elettroliti, e queste differenze sono possibili in quanto le strutture che li separano non sono liberamente permeabili a tutte le sostanze. Proprio da questa semipermeabilità delle membrane biologiche scaturisce il problema della pressione osmotica.

Infatti, tutti i liquidi dei vari compartimenti dell'organismo sono in equilibrio osmotico tra loro: questo garantisce la stabilità delle strutture le cui pareti si comportano come membrane semipermeabili. Quindi, l'immissione nel plasma di una soluzione ipertonica ha notevoli conseguenze quali la fuoriuscita di acqua dai globuli rossi che raggrinziscono, lo scompaginamento delle cariche elettrostatiche di membrana che tengono distanziate tra loro le cellule (per cui c'è possibilità di impilamento trasversale degli eritrociti), richiamo di acqua dai tessuti con iniziale aumento di volume ematico; i vasi periferici in conseguenza si dilatano ed il flusso ematico aumenta in maniera considerevole così da causare una sensazione di calore. Per compenso, la gettata cardiaca aumenta, e quindi il cuore è costretto ad un maggior lavoro. Questo richiamo di acqua dai tessuti, comporta modificazioni a livello delle cellule; perdita di acqua, neoformazione di particelle osmotiche (o reflusso di sodio), reingresso di acqua con iperidratazione, ed, infine, ritorno alle condizioni di normalità.

La perdita di acqua dai tessuti può causare dolore ed alcuni sistemi cellulari particolarmente delicati, quali il sistema nervoso centrale, possono addirittura andare incontro ad alterazioni funzionali rilevabili clinicamente (irrequietezza, convulsioni).

\section{Principali effetti collaterali dei mezzi di contrasto}

La somministrazione di mezzi di contrasto può, a volte, indurre effetti collaterali indesiderati, con meccanismi differenti e con manifestazioni cliniche diverse.

\section{1) Reazioni allergiche}

La maggior parte degli Autori $(2,6)$ correla le reazioni allergiche all'iper reattività di alcuni individui verso sostanze specifiche; tale reazione può essere dovuta ad una dimostrabile interazione tra antigene ed anticorpo, cioè essere tipica reazione anafilattica, oppure, pur assumendone le caratteristiche, non consentire l'individuazione di un meccanismo antigene-anticorpo (ed essere pertanto di tipo anafilattoide) oppure, ancora, estrinsecarsi con manifestazioni abnormi di idiosincrasia, per cui un soggetto risponde a dosi normali di un preparato come nei confronti di dosi tossiche.

\section{2) Reazioni immunologiche}

La presenza di un meccanismo immunologico alla base dei fenomeni di reattività abnorme al mezzo di contrasto è tutt'ora controversa, anche se recenti stu- di (30) hanno dimostrato l'esistenza di anticorpi specifici.

La maggior parte delle reazioni ai medicamenti è causata da un ristretto gruppo di allergeni, sebbene quasi tutti i farmaci possano agire come tali, lo Iodio tra questi. I composti iodati hanno la possibilità di combinarsi con le proteine e quindi possiedono la potenzialità di agire come apteni. Le proteine tessutali che si liberano anche per un lieve traumatismo locale assumono il ruolo di antigene ausiliario ed a contatto con il mezzo di contrasto introdotto, che funge da aptene, formano un antigene completo. Perché avvenga la reazione scatenante, basta che l'organismo venga successivamente a contatto con il solo aptene. Dal punto di vista clinico tale meccanismo immunologico può essere comprovato dal tipo di reazioni allergiche che possono comparire dopo esami radiologici: dall'orticaria alla vasculite leucociclastica cutanea, fino alle reazioni di tipo sistemico quali asma bronchiale, shock anafilattico, porpora trombocitopenica, etc. Un dato a favore della teoria immunologica è il fatto che le reazioni abnormi si verificano più facilmente in soggetti con anamnesi allergica positiva agli stessi mezzi di contrasto, o in quei soggetti con una storia di precedenti reazioni allergiche in generale.

Per quanto riguarda lo specifico meccanismo che scatena le reazioni di intolleranza immunologica, numerosi studi sugli animali e sull'uomo (28) hanno dimostrato che i mezzi di contrasto sono degli attivatori del complemento.

In vitro è stato rilevato inoltre che, dopo incubazione di siero normale con mezzo di contrasto, si osserva un'attivazione del complemento dipendente dalla dose, dal tempo di esposizione e dalla temperatura; il siero così trattato manifesta un'attività chemiotattica che depone a favore della liberazione di tossine anafilattiche. Queste tossine, tuttavia, hanno anche la capacità di liberare istamina dalle mastcellule e dai leucociti basofili. In effetti, l'iniezione endovenosa di quasi tutti i mezzi di contrasto provoca, entro pochi minuti, un raddoppiamento del livello plasmatico di istamina, che torna a valori di partenza dopo circa 20 minuti. Il potere istaminoliberatore è, secondo alcuni Autori, correlato alla velocità di iniezione, per cui la liberazione di istamina diminuirebbe, o sarebbe del tutto assente, se viene diminuita la velocità di sommi- 
TAB. I

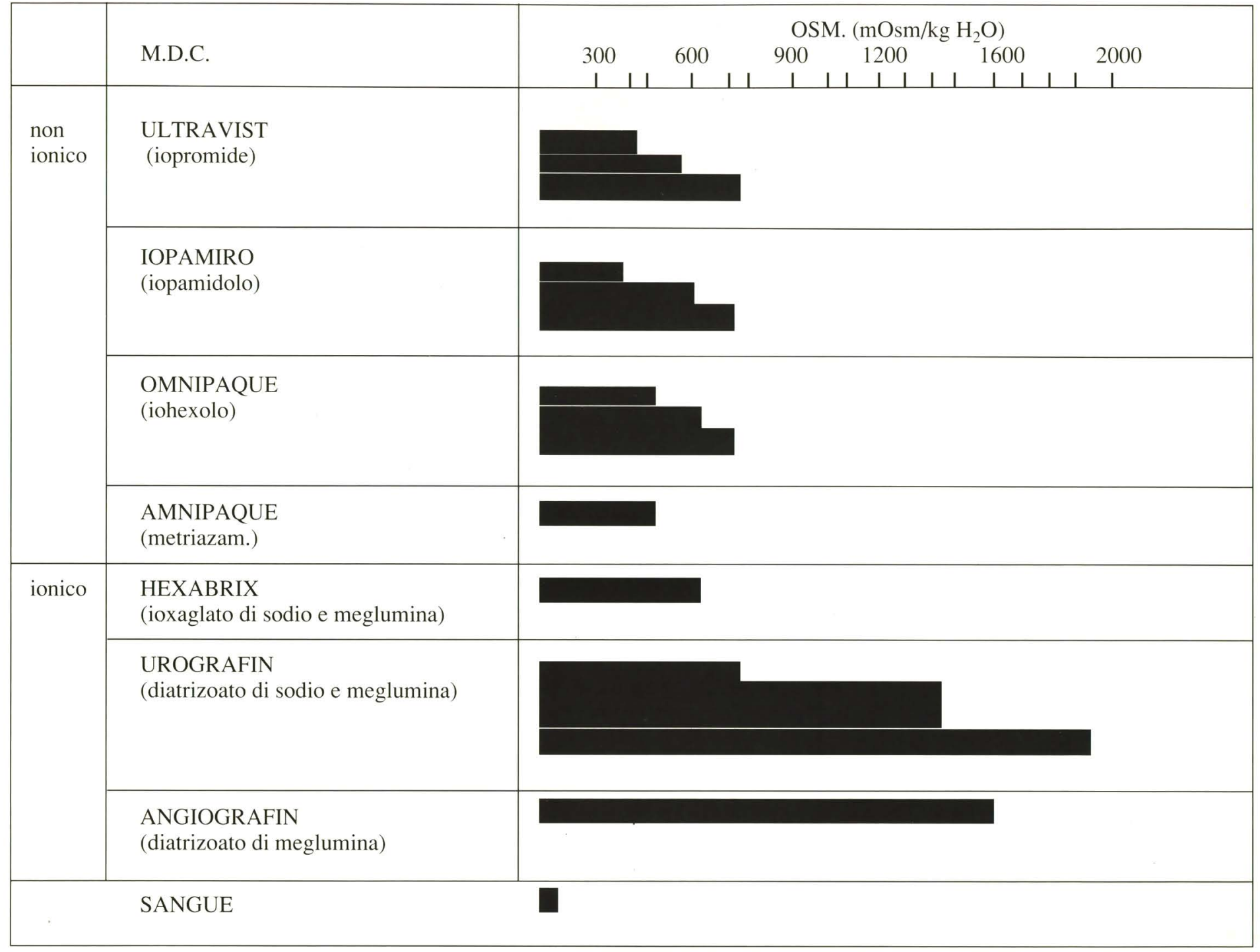

nistrazione del mezzo di contrasto.

Altri studi (35) hanno dimostrato che, accanto all'attivazione del complemento ed alla liberazione di istamina, ci può essere un effetto dei mezzi di contrasto sul sistema della coagulazione intravascolare; queste sostanze possono determinare l'attivazione delle funzioni piastriniche e si è evidenziata la comparsa di prodotti di degradazione della fibrina nel siero. In vitro, d'altra parte, è stata constatata inibizione dell'aggregazione piastrinica indotta da trombina, A.T.P. e adrenalina; allungamento del tempo di trombina; liberazione di serotonina dalle piastrine con modificazioni morfologiche delle stesse. In vivo l'effetto anticoagulante è comunque modesto anche se può persistere per 24 ore dopo l'esa- me contrastografico.

3) Reazioni da tossicità indiretta, cioè correlabili alle azioni farmacologiche delle molecole presenti nel mezzo di contrasto, possono essere attribuite sia al catione (Sodio o Meglumina) che all'anione iodato in toto che alle quantità, anche se minime, di Iodio liberate dall'anello benzenico. L'alterazione dell'integrità della barriera ematoencefalica connessa all'iperosmolarità dei mezzi di contrasto, ma secondo alcuni Autori (58, 59) invece, dovuta alla solubilità del mezzo nei lipidi, determina il passaggio, attraverso le membrane delle cellule endoteliali cerebrovascolari, del mezzo di contrasto dal circolo ematico nel tessuto nervoso centrale con conseguenti mani- festazioni neurotossiche quali convulsioni, paralisi e coma. Anche l'uso dei mez. zi ionici, a motivo della presenza di cariche elettriche, può comportare alterazioni nella trasmissione degli impulsi nervosi basati, notoriamente, su scambi ionici. In casi rarissimi e sempre in correlazione con un deficit escretorio, il mezzo di contrasto può persistere nell'organismo per periodi prolungati così che il legame iodio-benzenico, se pur altamente stabile, può essere metabolizzato a livello epatico con conseguente liberazione di Iodio libero in quantità minime, ma sufficienti a indurre qualche effetto biologico indesiderato. Alcuni effetti tossici dei mezzi di contrasto sono stati anche messi in relazione con la regione cationica della molecola, con riferimento in 
special modo a Sodio e Meglumina, che sono i più largamente utilizzati nella formazione di sali.

\section{4) Reazioni correlate alla osmolarità} Un aumento della osmolarità extracellulare, come avviene dopo l'iniezione di soluzioni di mezzo di contrasto ionico, causa una fuoriuscita di liquido intracellulare verso il compartimento extracellulare nel tentativo di ristabilire l'equilibrio osmotico. Questo movimento di liquidi conduce ad un aumento della viscosità interna delle cellule esposte al mezzo di contrasto, che sono soprattutto quelle contenute nel sangue e l'endotelio dei vasi. Ne conseguono deformazioni e irrigidimento degli eritrociti, distacco dei legami intercellulari a livello endoteliale, con libero passaggio di sostanze che normalmente non raggiungono i tessuti. Tale effetto sui legami intercellulari può dare ripercussioni soprattutto a livello della barriera ematoencefalica; una eventuale alterazione della integrità di questo apparato di difesa può infatti condurre a fenomeni di neurotossicità, dovuti al passaggio del mezzo di contrasto nel tessuto cerebrale.

L'iperosmolarità conduce anche a reazioni emodinamiche quali ipervolemia, in quanto vi è richiamo di liquidi dai tessuti al lume vasale, con aumento del volume ematico e del flusso ematico proprio in corrispondenza della sede di iniezione; questo fatto è responsabile della sintomatologia dolorosa che accompagna frequentemente la arteriografie periferiche specialmente all'avambraccio, carotide esterna ed arti inferiori. Il mezzo di contrasto ideale dovrebbe essere isotonico col sangue, cioè dovrebbe avere un'osmolarità di 300 mOsm; sebbene nessun mezzo di contrasto soddisfi tale caratteristica, i mezzi di contrasto di nuova generazione non ionici (Iopamidolo, Iopramide) e ionici (Ioxaglato) presentano una minore osmolarità rispetto ai meno recenti (Diatrizoati) e quindi una maggiore tollerabilità $(32,48,62)$ vedi Tabella I.

\section{Nefrotossicità dei mezzi di contrasto iodati}

Negli ultimi decenni i mezzi di contrasto iodati hanno rappresentato una delle cause più frequenti di insufficienza renale acuta. È stato infatti stimato (29) che il $10 \%$ dei casi di insufficienza renale acuta è il risultato della somministrazione di mezzi di contrasto iodati, e che questi agenti sono causa di insufficienza renale nei soggetti ospedalizzati quanto gli aminoglicosidi (22).

La nefropatia da mezzo di contrasto sembra interessare il $12 \%$ circa dei pazienti trattati: esiste tuttavia un'ampia variazione delle percentuali riportate $(10,13,17,18,20,26,39,41,43,45$, $49,50,53,55,57,60,61)$, dovuta alla scarsa omogeneità delle popolazioni studiate ed alla arbitrarietà dei valori fissati per definire l'insufficienza renale acuta (19), la cui incidenza sembra essere in continuo aumento a motivo della crescente richiesta di esami contrastografici, quali la tomografia computerizzata e la angiografia digitalizzata.

Numerose condizioni sono implicate come possibili fattori di rischio per l'insorgenza della nefropatia da mezzo di contrasto, e includono: età del paziente, insufficienza renale preesistente $(7,9,10)$, diabete mellito $(20,24,41,57)$, mieloma multiplo e discrasie plasmacellulari (4, 37,50 ), anemia (61), proteinuria ( 53), stati di disidratazione $(3,24,25)$, esposizione concomitante ad altri agenti nefrotossici (1), ripetute esposizioni a mezzi di contrasto in giorni ravvicinati $(1,7$, $10,25)$, iperuricemia $(7,20)$, il fatto che la somministrazione sia intraarteriosa piuttosto che endovenosa (61), presenza di scompenso cardiaco (54), presenza di ipertensione arteriosa con lesioni nefroangiosclerotiche $(9,25)$, trapianto renale (55).

L'esistenza, dunque, di un'alterazione della funzionalità renale precedente l'iniezione di mezzo di contrasto, favorirebbe l'insorgenza di una insufficienza renale acuta come è stato evidenziato da più Autori $(11,29,47,52)$, a motivo di un eccessivo carico osmotico per i nefroni superstiti funzionanti, e conseguenti lesioni anatomiche a livello delle cellule dell'epitelio del tubulo prossimale.

Nei pazienti diabetici l'incidenza di insufficienza renale acuta è direttamente proporzionale all'incremento della creatinina sierica basale; infatti i pazienti diabetici con una creatinina sierica maggiore di $1,5 \mathrm{mg} / \mathrm{dl}$ hanno circa il $50 \%$ di probabilità di sviluppare un'insufficienza renale acuta da mezzi di contrasto, che nel $56 \%$ dei casi si rivela irreversibile. Inoltre, Shieh et al, hanno riportato come i pazienti con Diabete mellito tipo II siano meno esposti alla insorgenza della insufficienza renale acuta rispetto ai pazienti con Diabete mellito tipo I.

Riguardo alla patogenesi dell'aumentato rischio di nefropatia da mezzo di contrasto, si ipotizza la presenza nelle molecole contrastografiche di fattori che in qualche modo contribuiscono all'aumento della viscosità sanguigna, all'ipossia post-capillare con formazione di microtrombi, e ad un'alterazione della deformabilità degli eritrociti; l'aterosclerosi e la glomerulopatia diabetica, la compromissione vascolare secondaria all'ipertensione, complicherebbero e completerebbero il quadro. La nefropatia indotta da mezzi di contrasto iodati è stata valutata mediante la determinazione dei livelli sierici di creatinina ed azotemia (5, $36)$, la clearance della creatinina $(5,36)$, e l'insorgenza di oliguria (55).

Dato che i livelli di creatinina sierica possono salire già 24 ore dopo l'esposizione al mezzo di contrasto e raggiungere il picco dal $3^{\circ}$ al $4^{\circ}$ giorno, la misurazione dei livelli di creatinina sierica nel $5^{\circ}$ giorno dopo l'esposizione al mezzo di contrasto è il metodo più pratico per identificare la nefropatia da mezzo di contrasto (42), come anche l'incremento dei valori di creatinina al di sopra del $50 \%$ del valore basale, o un incremento di $1 \mathrm{mg} / \mathrm{dl}$; oliguria è presente in circa il $30 \%$ dei casi, e dura dai 2 ai 5 giorni, con recupero entro il $7^{\circ}$ giorno. Il tempo trascorso tra l'oliguria e l'innalzamento della creatinina sierica dipende dal valore basale di creatinina prima di ricevere il contrasto. La tossicità renale sembra' essere favorita da insufficienza epatica coesistente. Infatti il sistema epatico e renale rappresentano le maggiori vie di escrezione degli agenti contrastografici impiegati per la colecistografia orale e per la urografia endovenosa, per cui una ridotta coniugazione epatica induce necessariamente un aumento della frazione escreta dal rene. Quando è presente un danno renale più serio il tempo di recupero è in genere più prolungato. L'insufficienza renale acuta può essere non oligurica, più comunemente in pazienti che presentano livelli di creatinina sierica più bassi precedentemente all'esame contrastografico. Occasionalmente l'insufficienza renale acuta è irreversibile e necessita di un trattamento dialitico sostitutivo a lungo termine.

Per quanto riguarda il meccanismo re- 

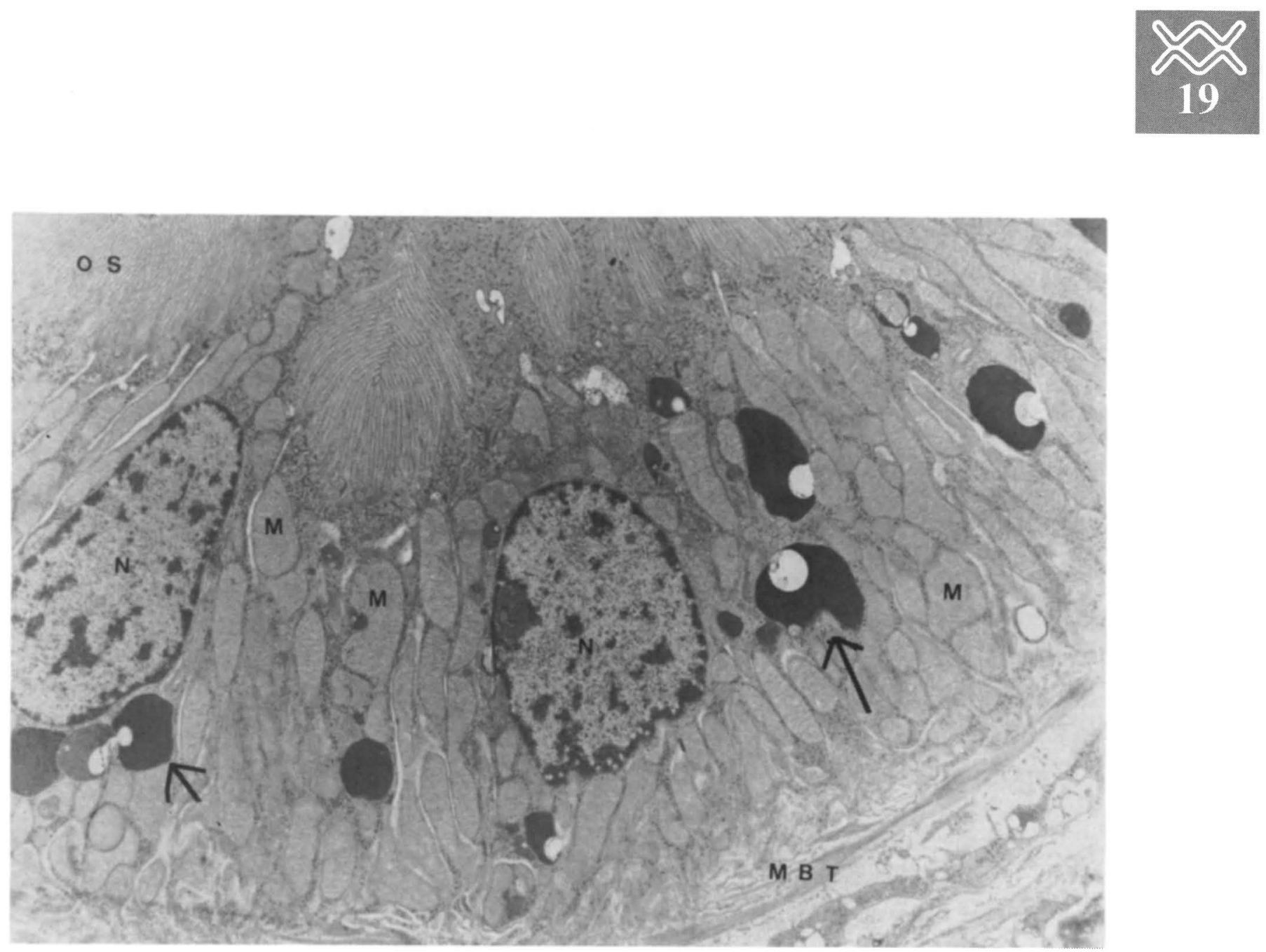

Fig. 1 - Microscopia elettronica: $5800 X$

Tubulo prossimale di ratto Wistar outhbred trattato con mezzo di contrasto iodato non ionico (Iopamidolo 370 ), senza evidenti alterazioni cellulari.

$M B T=$ membrana basale tubulare

$N=$ nucleo della cellula epiteliale

$O S=$ orletto a spazzola

$M=$ mitocondri

Le frecce evidenziano corpi lisosomiali

sponsabile dell'insufficienza renale, tutti gli Autori sono concordi nel riferire 5 cause fisiopatologiche:

\section{1) Interferenza con la perfusione renale:} l'iniezione del mezzo di contrasto provoca nel circolo ematico una transitoria e breve $(10,20 \mathrm{sec})$ vasodilatazione cui segue una vasocostrizione che riduce il flusso sanguigno renale per un periodo di tempo di qualche ora, variabile a seconda della dose impiegata. La risposta emodinamica è inoltre chiaramente dipendente dall'osmolarità della soluzione, tanto che i mezzi di contrasto a bassa osmolarità inducono una vasocostrizione di modesta entità, rispetto a quelli a più alta osmolarità. Per quanto riguarda la genesi della vasocostrizione da mezzo di contrasto vi sono varie ipotesi.

Secondo Deray e coll. (1990) l'iniezione di mezzo di contrasto provocherebbe una modificazione nella composizione del- l'urina del tubulo contorto distale avvertita dalla macula densa tramite un mediatore, l'adenosina. La macula densa trasmetterebbe poi alla arteriola afferente glomerulare un messaggio responsabile della modificazione delle resistenze arteriolari.

Questa ipotesi sarebbe confermata dal fatto che la teofillina (antagonista dei recettori per l'adenosina) diminuisce la vasocostrizione se somministrata prima dell'iniezione del mezzo di contrasto; viceversa il dipiridamolo (antagonista del "reuptake" dell'adenosina) l'accentuerebbe.

Altri Autori $(8,27)$, invece, dimostrano che la transitoria vasodilatazione che segue l'iniezione di mezzo di contrasto viene antagonizzata da una fisiologica stimolazione del sistema renina-angiotensina, con conseguente vasocostrizione sistemica e diminuizione del flusso sanguigno renale; ciò non sarebbe, di per sé, sufficiente a provocare uno stato di preischemia, ma la dimostrazione di una ridotta sintesi di prostaglandine durante l'iniezione con mezzo di contrasto spiegherebbe, invece, come la coesistenza di questi due fattori (induzione di renina e blocco della produzione di prostaglandine) sia in grado di provocare uno stato di pre-ischemia e pre-insufficienza renale (56), che i già citati fattori di rischio convertirebbero in insufficienza renale acuta.

2) Alterata permeabilità selettiva glomerulare: i mezzi di contrasto sembrano alterare la distribuzione di cariche elettriche lungo la membrana di filtrazione glomerulare e così promuovere il passaggio di proteine dal lume capillare allo spazio urinario glomerulare (38); questo massivo flusso di proteine nei tubuli potrebbe favorire un danno tubulare indiretto. 
3) Ostruzione tubulare: un altro meccanismo patogenetico è l'ostruzione meccanica dei tubuli renali da parte di proteine urinarie e cristalli di urato. Infatti, i mezzi di contrasto, e specialmente quelli usati nella colecistografia per via orale, sono potenti agenti uricosurici.

Alcune osservazioni (Hearly et al., 1963; Rees et al., 1965) suggeriscono, inoltre, che i mezzi di contrasto sono in grado di accelerare la precipitazione della proteina di Bence Jones, e la formazione di cilindri proteič intratubulari come è noto avvenire in caso di discrasie plasmacellulari.

4) Reazioni immunologiche: Ring et al (1978) hanno dimostrato reazioni di ipersensibilità nel coniglio dopo iniezioni di mezzi di contrasto iodati con rilascio di istamina e serotonina.

Un'altra ipotesi attribuisce queste reazioni di ipersensibilità ad anticorpi IgM-anti mezzo di contrasto; si verrebbero così a formare degli immunocomplessi circolanti che promuovono il rilascio di sostanze vasoattive causando ischemia renale.

5) Danno tubulare diretto: dopo somministrazione endovenosa, solo una piccola quota del mezzo di contrasto iodato si lega all'albumina plasmatica o alle alfa globuline, e circa il 5-10\% della sostanza viene captata dagli eritrociti; il resto della dose somministrata viene escreto a livello renale per lo più attraverso la filtrazione glomerulare.

Il mezzo di contrasto durante il transito attraverso il tubulo renale non subisce alcuna modificazione ad eccezione di un aumento della propria concentrazione dovuta al riassorbimento tubulare di acqua e soluti, in particolare da parte del tubulo prossimale. Danni tubulari diretti possono aversi per uptake del mezzo di contrasto all'interno delle cellule epiteliali tubulari o per lesione da iperosmolarità del mezzo di contrasto.

Cunningam et al (1974), hanno dimostrato che i mezzi di contrasto ionici, quali Diatrizoato e Iothalamato alterano il trasporto tubulare di Sodio, forse inibendo l'attività dell'enzima $\mathrm{Na}-\mathrm{K}$ ATPasi.

Humes et al (1987) hanno invece valutato la tossicità diretta di Diatrizoato sodico e Meglumina attraverso isolamento ed incubazione per $90 \mathrm{~min}$ delle cellule del tubulo contorto prossimale con la sostan- za contrastografica; si evidenziava una significativa diminuzione del contenuto tubulare in potassio e un altrettanto significativo incremento del contenuto di calcio.

Lasser et al (1974), hanno studiato gli effetti sul piano istologico di diversi mezzi di contrasto iodati iniettati direttamente nei reni di cani. I risultati hanno dimostrato che la porzione tossica della molecola è la parte alogena, dal momento che l'iniezione della sola porzione non alogena provoca danni istologici modestissimi e peraltro non specifici in quanto riscontrabili anche dopo iniezione di soluzioni ipertoniche di altro tipo (34), localizzati soprattutto a livello dei tubuli contorti prossimali (31) e consistenti in intensa vacuolizzazione a carico delle cellule tubulari prossimali.

Relativamente alla presunta minor nefrotossicità dei nuovi mezzi di contrasto a bassa osmolarità non ionici, la disputa è ancora aperta. In studi comparativi tra mezzi di contrasto ionici e non ionici $(14,33,51)$ si è dimostrato che dopo arteriografia renale i mezzi di contrasto iodati di tipo non ionico causano minor variazioni nella permeabilità glomerulare, nella filtrazione e circolazione glomerulare, rispetto agli ionici monomeri e, globalmente, minori modificazioni della funzione renale (Fig. 1)

Inoltre, sebbene i mezzi di contrasto iodati non ionici raggiungano una concentrazione intratubulare più elevata rispetto ai mezzi di contrasto iodati di tipo ionico, essi presentano anche una minore tossicità cellulare tubulare. Tali osservazioni portano a concludere che nei pazienti con normale funzione renale il rischio di nefropatia da mezzi di contrasto è modesto a prescindere dal mezzo di contrasto utilizzato, mentre nei pazienti che presentino i già citati fattori di rischio, l'insorgenza di insufficienza renale risulta statisticamente più rilevante con l'utilizzo dei mezzi di contrasto iodati di tipo ionico.

\section{BIBLIOGRAFIA}

1. Alexander RD, Bekes SL, Abuelo JG. Contrast media induced oliguric renal failure. Arch Intern Med 1978; 138: 381-4.

2. Ansell G. Adverse reactions to contrast agents. Invest Radiol 1970; 5: 374 .

3. Ausar Z, Baldwin DS. Acute renal failure due to radiocontrast agents. Nephron 1976; 17: 28-40.

4. Berdon WE, Schwartz RH, Becker J, Baker DH. Tamm-Horsfall proteinuria: its relationship to prolonged nephrogram in infants and children and to renal failure following intravenous urography in adults with Multiple Myeloma. Radiol 1969; 92: 714-22.

5. Berkseth RO, Kjellstrand CM. Radiologic contrast induced nephropaty. Med Clin North Am 1984; 68: $351-70$

6. Brasch RC. Allergic reactions to contrast media. Accumulated evidence. Amer J Roentgenol 1980; 134: 797.

7. Byrd L, Scherman RL. Radiocontrast-induced acute renal failure: a clinical and phatophisiologic review. Medicine 1979; 58: 270-9.

8. Caldicott WJH, Hollemberg NK, Abrams HL. Caracteristics of response of renal vascular bed to contrast media. Evidence of vasocostrition induced by renin-angiotensin system. Invest Radiol 1970; 5: 539.

9. Carvallo A, Rakowsky TA, Angry WP, Schreiner GE. Acute renal failure following drip infusion pyelography. Am J Med 1978; 65: 38-45.

10. Cochran ST, Wong WS, Roe DJ. Predicting angiography induced acute renal function impairment. Clinical risk model. Am J Radiol 1983; 141: 1027. 
11.

Conin RE. Southwestern internal medicine conference. Renal failure following radiologic procedures. The Am $\mathbf{J}$ of the medical sciences $1989 ; 298,5$ : 342-56.

12. Cunningham JJ, Friedland GW, Thurber B. Immediate diuretic effects of intravenous sodium diatrizoate in injection. Radiology 1974; 11: 85 .

13. D'Elia JA, Gleason R, Alday $M$, et al. Nephrotoxicity from angiographic contrast material. A prospective study. Am J Med 1982; 72 : 719.

14. Denys BG, Reddy PS, Uretsky BF. Nephrotoxicity of a non ionic (Iopamidol), versus an ionic (Diatrizoate) contrast agent in the patient after cardiac transplant with moderate cyclosporine. Induced renal insufficiency. Cardiology 1989; 64: 405-6.

15. Deray G, Dubois M, Baumelou B, Martinez F, et al. A comparison of the nephrotoxicity of diatrizoate and ioxaglate in rats. I.C.R., Paris; juillet 1989; 342 .

16. Deray G, Baumelou B, Dubois M, Baumelou A, Jacobs C. Phisiopathologie de la nephrotoxicité des produits de contraste iodés. J Radiol 1990; 71, 1: 1-3.

17. Diaz Buxo JA, Wagonen RD, Hatterry RR, et al. Acute renal failure after excretory urography in diabetic patients. Am Intern Med 1975; 83: 155 .

18. Eisemberg RL, Bank WO, Hedgcock MW. Renal failure after major angiography can be avoided with hidratation. Am J Radiol 1981; 136: 859.

19. Gomes AS, Baker JD, Martin Paredero V, et al. Acute renal dysfunction after major arteriography. Am J Radiol 1985; 145: 1249.

20. Harkonen S, Kjellstrand CM. Intravenous phyelography in non uremic diabetic patients. Nephron 1979; 24: 268.

21. Hearly JK. Acute oliguric renal failure associated with multiple myeloma. Report of three cases. MJ 1963; 1: 1126-30.

22. Hou S, Buscinsky DA, Wish JB, et al. Hospital acquired renal insufficiency: a prospective study. Am J Med 1983; 74: 243-8.

23. Humes HD, Hunt DA, White MD. Direct toxic effect of the radiocontrast agent diatrizoate on renal proximal tubule cells. Am J Phisiol 1987; 252: 246.

24. Kamdar A, Wiedmann P, Makoff DL, Massry SG. Acute renal failure following intravenous use of radiographic contrast dyes in patiens with diabete mellitus. Diabetes 1977; 26: 643-9.

25. Krumlovsky FA, Simon N, Santhanam S, Del Greco F, Roxe D, Pomarane MM. Acute renal failure. Association with administration of radiographic contrast material. Jama 1978; 239, 5: 128.

26. Kumar S, Hull JD, Lathy SI, et al. Low incidence of renal failure after angiography. Arch Int Med 1981; 141: 1268.

27. Larson TS, Huston K, Mertz JI, Romero JC, Knox FG. Renal vasocostrictive response to contrast medium. The role of sodium balance and the renin-angiotensin system. J Lab Clin Med 1983; 101: 385-91.

28. Lasser EC, Kolb WP, Lang JH. Contrast media activation of serum complement system. Invest Radiol 1974; 9, 4: 6A.

29. Lautin et al. Radiocontrast associated renal dysfunction: incidence and risk factors. AJR July 1991; 157: 49-58.

30. Lolli AF. Contrast media reactions: data anlysis and hipotesis. Radiology 1980; 134: 1.

31. Messana JM, Cresliusky DA, Nguyen VD, Humes HD. Comparision of the toxicity of the radiocontrast agents, iopamidal and triazoate, to rabbit renal proximal tubule cells in vitro. J Pharmacol Exper Ther 1988; 244: 1139-44.
32. Miklautz H. Fichte K, Wegscheider K. Osmolarity of non ionic contrast media. In: Recent developments in non ionic contrast media, Ed. by V. Taenzer, S. WendeThieme, Stuttgard 1989; 16-20.

33. Moore RD, Earl P Steinberg, Neil R Powe et al. Nephrotoxicity of high-osmolarity versus lowosmolarity contrast media: randomized clinical trial. Radiology 1992; 182: 649-55.

34. Moreau JF, Druz D, Sabto J, Jerugers P, Kleuknecht D, Hiuglais N, Michel JR. Osmotic nephrosis induced by water soluble tri-iodinated contrast media in man. Radiology 1975, 115: 329-36.

35. Mosier LD, Joist JH, Chance $\mathrm{D}$, et al. In vitro effects of ionic and nonionic contrast media on coagulation, platelet function and fibrinolysis. Present at the 73rd meeting of the radiological society of North America, Chicago, Il. Nov 1987; 30.

36. Mudge GH. Nephrotoxicity of urographic radiocontrast drugs. Kidney Int 1980; 18: 540-52.

37. Myers GH, Witten DM. Acute renal failure after excretory urography in Multiple Myeloma. Am J Roentgen 1971; 113: 583-8.

38. Nicot GS, Merle LJ, Charmes JP, Valette JP, Novaille YD et al. Transient glomerular proteinuria, enzymuria and nephrotoxic reaction by radiocontrast media. JAMA 1984; 252: 2432-4.

39. Paredero MV, Dixon SM, Baker JD, et al. Risk of renal failure after major angiography. Arch Surg 1983; $118: 1417$.

40. Parfrey PS, Griffiths SM, Barrett BJ, Paul MD, Genge M et al. Contrast material induced renal failure in patients with diabetes mellitus, renal insufficiency, or both. N Engl J Med 1989; 320: 143.

41. Port FK, Wagoner RD, Fulton RE. Acute renal failure after angiography. Am J Roentgenol 1974; 121: 544. 
42. Porter GA. Contrast associated nephropaty. Am J Cardiol 1989; 64: 22E-26E.

43. Rahimi A, Edmonson RPS, Jones NF. Effect of radiocontrast media on the kidneys of patients with renal disease. Br Med J (Clin Res) 1981; 282: 1494.

44. Rees ED, Waugh WH. Factors in renal failure in multiple myeloma. Arch Int Med 1965; 116: 400-5.

45. Reiss MD, Bookstein JJ, Bleifer KH. Radiology aspects of renovascular hypertension. JAMA 1972; 221: 375 .

46. Ring J, Endrich B, Intaglietta M. Histamine release, complement consumption and microvascular changes after radiographic contrast media infusions in rabbits. J Lab Clin Med 1978; 92: 584-94.

47. Roo SR, Miezda MA, Leiter E. Renal failure in diabetes after interavenous urography. Urology 1980; 15: 577-80.

48. Schrader R, Baller D, Hoefte A, Korb H, Wolpers HG, Hellige G. Reduced side effects of low osmolarity non ionic contrast media in coronary arteriography, comparative experimental study in dogs. In: Contrast media in urography, angiography and computerized tomography. Ed by Taenzer V, Zertler E, Thieme, Stuttgard 1983; 67-77.

49. Shafi T, Chon SY, Porush JG, et al. Infusion pyelography and renal function: effects in patients with cronic renal insufficiency. Arch Int Med 1978; 138: 1218.

50. Shieh DS, Hirsh SR, Boshell $\mathrm{BR}$, et al. Low risk of contrast media induced acute renal failure in non azotemic type 2 diabetes mellitus. Kidney Int 1982; 21: 739.

51. Stacul F, Carraro M, Magnaldi S, Faccini L, Guarnieri G, Dalla Palma L. Contrast agent nephrotoxicity: comparison of ionic and non ionic contrast agents. AJR 1987; 149: 1287-9.
52. Sven-Ola Hietala, Almen T. Patient risk factors of importance in nephrotoxicity of contrast media. 1989 Elsevier Science publishers BV (Biomedical division) 121-30.

53. Swartz RD, Rubin JE, Leeming BW et al. Renal failure following major angiography. Am J Med 1978; 65: 31.

54. Taliercio CP, Vliestra RE, Fisher LD, Burnett JC. Risk for renal dysfunction with cardiac angiography. Am J Med 1986; 104: 501-4.

55. Teruel JL, Marcen R, Onaindia $\mathrm{JM}$, et al. Renal function impairment caused by intravenous urography. Arch Int Med 1981; 141: 1271.

$56 . \quad$ Todavarthy SM, Castaneda W, Amplatz K. Redistribution of renal blood flow caused by contrast media. Radiology 1977; 122: 343-8.

57. Van Zee BE, Hoy WE, Talley $\mathrm{TE}$, et al. Renal injury associated with intravenous pyelography in non diabetic and diabetic patients. Am Intern Med 1978; 89: 51.
58. Waldron RL, Bridenbaugh R, Purkerson M, Dempsey EW. The effect on angiographic contrast media at the cellular level in the brain. Radiology 1973; 108: 187.

59. Waldron RL, Bryan- RN. Effects of contrast agents on the blood brain barrier. Radiology 1975; 116: 195.

60. Webb JA, Reznek RH, Cattell WR, et al. Renal function after high dose urography in renal failure. Br J Radiol 1981; 54: 479.

61. Weinrauch LA, Healy RW, Leland OS, et al. Coronagraphy, agiography and acute renal failure in diabetic azotemic nephropaty. Ann Intern Med 1977; 86: 56.

62. Wolf KJ, Steidle B, Banzer D, Seyferth W, Keisser R. Comparative evaluation of low osmolar contrast media in (femoral) arteriography. In: Contrast media in Urography, Angiography and computerized Tomography. Ed by Taenzer V. and Zeitler E; Theme Verlag, Stuttgard 1983; 102-6. 